in der innigsten Gemeinschaft mit ihm ist die Heimstätte dieses Namens, er hat Gebetsklang, und fast nur in Gebeten finden wir ihn in der Schrift. Die philosophische Bildung des gräcisierenden Judenthums scheute sich vor dem Plastisch-Concreten, vor dem Gewaltsamen dieses Wortes; und so mufste denn der bildliche Ausdruck aufgelöst, dessen Geist verflüchtigt werden, ähnlich wie Luther's „Ein feste Burg“ in „Ein starker Schutz ${ }^{“}$ corrigiert wurde. Auch in den Schulen von Tiberias und Sura mangelte es an dem Verständnifs für die Eigenart des alterthümlichen Gottesnamens.

\title{
Noch einmal Sauls Königswahl und Verwerfung.
}

\author{
Von C. H. Cornill.
}

Im Jahrgang VIII dieser Zeitschrift S. 223 ff. hat mein lieber Freund Budde einen neuen Versuch, das Räthsel der Composition der Bücher Samuelis zu lösen, gebracht, welcher sich besonders eingehend mit meinen beiden diesem Gegenstande gewidmeten Arbeiten beschäftigt. Dafs ich mich mit dieser Abhandlung Budde's auseinandersetzen müsse, war selbstverständlich; anderweitige dringende Pflichten haben mich bis jetzt daran gehindert : doch hoffe ich, dals die Verzögerung der Sache nicht zum Schaden gereicht ist.

Budde selbst bemerkt, dafs sein Ergebnifs sich in wesentlichen Stücken mit dem meinigen berühre, in anderen davon abweiche und es berichtige. Ich möchte zunächst einen Punkt herausgreifen, bei welchem ich gleichfalls im Princip mit Budde übereinstimme, obwohl dieser es in Abrede stellt. Er bezeichnet S. 227 die Stellen 10, 25-27 und 11, 12-14 als harmonistische Klammern zur Ausgleichung von $G$ und $M$ (die Bedeutung dieser sehr einfachen 
und zweckmäfsigen Siglen setze ich als aus Budde's Abhandlung bekannt voraus) und sagt dann S. 228 „... die so höchst einfache Art ihrer Verbindung durch jene zwei Klammern hat er (d. h. Cornill) nicht gesehen." Wenn ich S. 118 den ganzen Vers 11, 14 von dem Redactor eingesetzt sein lasse als einen „Ausgleichungsversuch ${ }^{*}$ von Cap. 11 mit 10, $17 \mathrm{ff}$; wenn ich S. 129 constatire, dafs mit 10, 24 der Faden plötzlich abreilse und dals wir in den Versen 25 und 26-27 „Eingriffe des Redactors“ anzuerkennen haben, der hier die jüngere Erzählung in den Rahmen der älteren Quelle einpassen will : so habe ich allerdings das Wort „Klammern ${ }^{*}$ nicht gebraucht, aber die Sache deutlich bezeichnet, wenigstens bezeichnen wollen. Nur habe ich zu beweisen gesucht, dafs diese Klammern zum Theil aus echtem altem Ueberlieferungsstoffe hergestellt sind, und diesen meinen Beweis hat Budde nach einer Richtung selbst noch verstärkt. Ich war ausgegangen von 10, 25-27. Dals diese Verse so, wie sie dastehn, die Klammer zwischen den Cap. 10 u. 11 sein sollen, ist klar, eben so klar aber auch, dals sie diesen Zweck nicht erfüllen. Mich hatte zuerst ein ästhetischer Eindruck stutzig gemacht : die Worte 10, $26 \mathrm{~b}$ hatten auch mir „das Herz gerührt" und ich glaubte in ihnen und den von ihnen nicht zu trennenden Worten 27 a ein echtes altes Werkstück erkennen zu müssen, das aus einer andern Stelle herausgebrochen war, um hier in werthloses Geröll vermauert zu werden. Dieser ästhetische Eindruck wurde bestätigt durch die sachlichen Gründe, welche Budde S. 227 noch schärfer formulirt, als ich es auf S. 118 gethan hatte. Nun galt es, den ursprünglichen Platz jener Worte zu ermitteln. Ich erkannte in Cap. 11 Vers 8 eine verdächtige Stelle. Dafs dieser Vers in dieser Gestalt von dem ursprünglichen Verfasser von Cap. 11 geschrieben sei, konnte und kann ich nicht glauben und wird auch Budde nicht glauben, der auf dies für meine ganze Beweisführung höchst wichtige 
sachliche Moment gar nicht eingegangen ist. Und wenn sich nun gerade an der genannten Stelle, wo der Eingriff einer fremden Hand ganz unleugbar ist, jenes muthmalslich echte alte Werkstück 10,26 b. 27 a so passend einfügen lief's, dafs ich wohl kühnlich behaupten darf, kein Mensch würde, wenn wir sie hier läsen, bezweifeln, dafs hier auch die ursprüngliche Stelle dieser Worte gewesen sei : so erschien mir damit der Wahrscheinlichkeitsbeweis für die Richtigkeit meiner Annahme erbracht. Freilich kann ich nicht den Vers einfach umstellen; ich mufs ,an jeder Stelle etwas streichen " S. 228. Aber wenn ich die Wahl habe, ויצאו כאיש אחר für Flicke eines Ueberarbeiters zu halten, oder einen so significanten Ausdruck wie : „und es gingen mit ihm die Wackern, denen Gott das Herz rührte, aber die nichtsnutzigen Leute sprachen : Was wird der uns helfen ?" so bin ich keinen Augenblick im Zweifel, wie die Entscheidung ausfällt. Allein gerade den Umstand, dafs ich ᄁan jeder Stelle etwas streichen mufs", führt Budde als Hauptargument gegen mich ins Feld : das ויבודה ולא הביאו לו מנחה in 8.27 sei sicher kein Zusatz, und es reicht aus, den Versuch zu vereiteln“, S. 229. Hier mufs ich nun einen principiellen Gegensatz constatiren, den ich absichtlich so schroff wie möglich formulire, obwohl ich weifs, dafs Budde diese Formulirung selbst ablehnen wird : aber seine ganze Argumentation gegen mich wäre nur dann zwingend, wenn jene ihm hier zugeschobene Formulirung in ihrer ganzen Schroffheit wirklich seine Meinung wäre. Ich behaupte : So grob mechanisch, wie es nach dieser Ausführung Budde's scheint, so rein äufserlich nur mit Scheere und Kleister hat meiner festen Ueberzeugung nach kein hebräischer Redactor oder Diaskeuast gearbeitet, dals er einfach irgendwo in seiner Vorlage einen Satz oder einen Satztheil herausgeschnitten und denselben dann ohne jede Veränderung an einer anderen Stelle eingeklebt hätte. Und 
das meint auch Budde nicht, der in dieser Zeitschrift Jahrg. VII S. 161 selbst so schön und wahr von „der wechselvollen Wirklichkeit der Hergänge und der freien Selbstbestimmung der Verfasser" redet, welcher der Quellenkritiker nicht Gewalt anthun dürfe. Wir beide stimmen darin überein, dafs die Verse 10, 25-27 die Klammer zwischen $M$ und $G$. sein sollen, wir beide stimmen darin überein, dafs die Verse hierzu "nicht genügen", dafs sie diesen Zweck nicht erfüllen : da glaube ich nun, diese Thatsache erklärt sich am Einfachsten und Natürlichsten, wenn der Redactor jene Klammer eben nicht ganz frei geschmiedet, sondern sie tant bien que mal aus einem echten Stücke der ihm vorliegenden Ueberlieferung zurechtgehämmert hat. Es entspricht das zudem auch vollständig dem anerkannten Conservatismus der hebräischen Diaskeuasten, welche sich in Zusätzen die grölste Freiheit nehmen, während sie sich nur schwer entschliefsen können, irgend ein Stück ihrer Vorlage völlig zu opfern, und lieber zu den gewaltsamsten Verrenkungen greifen, um einen vielleicht nicht einmal wesentlichen $\mathrm{Zug}$ zu retten. Dafs es bei einem solchen Verfahren nicht ganz glatt abgegangen ist, dafs schon der Diaskeuast an jeder Stelle eingegriffen hat, um die durch seine Manipulation entstehenden Risse und Fugen zu verputzen, und dafs wir daher, um das Ursprüngliche herzustellen, ${ }^{\text {an }}$ jeder Stelle etwas streichen müssen", ist ein so natürlicher und selbstverständlicher Vorgang, dafs ich ihn nicht als Gegengrund gegen meine Aufstellungen anerkennen kann. Und wenn Budde meint, dafs v. 27 b „sicher kein Zusatz" ist, wohl weil ihm der Ausdruck zu originell erscheint, so verweise ich ihn auf II Chron. 17, 5. Hier geben dem in der Chronik besonders verherrlichten Josaphat nicht etwa überwundene Feinde, bei welchen das Darbringen einer מנחה Dutzende von Malen vorkommt, sondern die eigenen angestammten Unterthanen beim Regierungsantritte Huldigungsgeschenke 
ויתנו כל יהודה טנחה ליהושפט, und der Chronist ist doch ein Autor, dessen spätes Zeitalter feststeht und bei dem von schriftstellerischer Kraft und Originalität nur in sehr beschränktem Malse die Rede sein kann. Die Redensart אשר עע אלהים בלבם dagegen, bei welcher ich vielleicht auch auf den Gottesnamen אלהים, nicht יהוה, aufmerksam machen darf, ist im Alten Testamente ganz ohne Parallele; die einzige ähnliche Stelle Jer. 4, 18 ist nach Construction und Sinn völlig andersartig. Ebenso kann ich in Bezug auf 11, 12-13 mich von Budde nicht für widerlegt halten. Ich hatte mit 10, 26 b. 27 a auch diese Verse in einer etwas veränderten Urgestalt für $G$ in Anspruch genommen und auf die Parallelstelle 14, $44-45$ verwiesen. Budde meint S. 229, dies Motiv liege sehr nahe und finde sich noch ähnlicher II Sam. 19, 23. Das Motiv ja; aber auch die stylistische Ausdrucksweise? 11, 13 wie 14, 45 wird die Nichtzulassung einer Todesstrafe motivirt durch eine ישועה בישראל, ein doch immerhin charakteristischer Ausdruck, von welchem sich II Sam. 19, 23 keine Spur findet; dals es 14, 45 שועה תשועה heifst, 11, 13 dagegen um so weniger verschlagen, als der Verfasser von $G$ in 11, 9 gleichfalls geschrieben hatte. Ich halte also in Bezug auf jene beiden Stellen 10, 26-27. 11, 12-13 meine alte Position voll und ganz aufrecht und freue mich, durch Budde selbst die sachlichen Gründe für jene meine Annahme zum Theil noch wesentlich schärfer und klarer ausgesprochen zu finden.

Wichtiger ist der zweite controverse Punkt. Ich hatte $\mathbf{M}$ für wesentlich überarbeitet erklärt und nur den Grundstock dieser Quellenschrift für $\mathrm{E}$ in Anspruch genommen; Budde leitet ganz $M$ von $E a b$, was ich in meiner zweiten $A b$ handlung gleichfalls schon als Möglichkeit ins Auge gefalst hatte, und behauptet, grade die von mir verworfenen Bestandtheile legten hierfür am lautesten Zeugnifs ab. Letzteres hat Budde in der That durchaus schlagend und 
überzeugend dargethan und ich stehe nicht an, in diesem Punkt ihm vollkommen beizustimmen. Aber ist damit meine ganze Position erschüttert? Ich glaube nicht. Budde selbst sagt : „Alles beruht hier auf dem sachlichen Moment der grundsätzlichen Verwerfung des Königthums, und vielleicht könnte man Cornill zustimmen, wenn der Rest der beiden Abschnitte diesem Moment entschieden widerspräche" S. 231. Letzteres habe ich behauptet und behaupte es noch. Betrachten wir einmal $M$ als ursprüngliche Einheit. Da mufs uns zunächst in Cap. 8 das gänzliche Fehlen des theokratischen Moments auffallen. Selbst wenn man die Worte v. 10 u. 11 ,da redete Samuel alle Worte Jahres zu dem Volke, welches von ihm einen König erbat, und sprach" so verstehn dürfte, dafs Samuel dem Volk vor der berühmten Königsrede auch noch mitgetheilt habe, was Jahve v. 7 u. 8 zu ihm geredet hatte, was hätte das Volk dann erfahren? Dafs Jahve von Anfang an ohne Weiteres dem Begehren zustimmt und dem Samuel sein Widerstreben als eine unberechtigte persönliche Empfindlichkeit verweist. Mufs man aber die fragliche Stelle so verstehn, dafs erst mit v. 11 die Rede Samuels an das Volk beginnt, so enthält dieselbe von theokratischen Gedanken und Momenten rein nichts, sondern weist darauf hin, dafs die von dem Volke begehrte Woblthat doch sehr ihre zwei Seiten habe und auch zur Plage werden könne - also eine Erwägung lediglich der äufserlichen Klugheit und Opportunität, die zudem noch durch die von mir angeführte Parallelstelle 22, 7 eine ganz eigenthümliche Beleuchtung erhält und so auch des letzten Scheins von specifisch theokratischem Charakter entkleidet wird. Als das Volk auch auf diese Gefahr hin bei seinem, nach v. 20 durchaus motivirten, Verlangen bleibt (die Worte 8,19 „Aber das Volk wollte nicht hören auf die Stimme Samuels" empfangen ihre Färbung durch den Inhalt des Vorhergehenden und sind für sich allein nicht genügend, 
um „die Mifsbilligung des Königthums durch Samuel und die Verstockung des Volkes ... in ihrer vollen Schärfe ${ }^{u}$ zu erweisen), wird die definitive Königswahl anberaumt. Um sein Gewissen zu salviren und seinen Standpunkt klarzustellen, redet Samuel noch einmal einige einleitende Worte 10, 18-19 und vollzieht dann die Königswahl unter persönlicher freudiger Anerkennung des durch das heilige Loos Erwählten und dem allgemeinen Jubel des Volkes. Und jetzt erst, nachdem die Sache geschehen ist und nicht mehr rückgängig gemacht werden kann, jetzt öffnet Samuel alle Schleufsen seiner homiletischen Beredsamkeit, überschüttet das Volk mit einer Fluth der schwersten Vorwürfe und sagt ihm ein Mal über das andere, welch eine unverzeihliche Sünde es begangen habe, und diese durch ein Wunder unterstützte Rede wirkt so mächtig, dafs aus der jubelnden Menge von 10, 24 eine zerknirschte und fassungslose Schaar wird 12, 19. Dafs hier eine ursprüngliche und einheitliche Conception vorliege, kann ich auch jetzt noch nicht glauben. Dann hätte Samuels principielle Stellung von Anfang an viel klarer motivirt und viel deutlicher ausgesprochen werden müssen, dann hätte vor allen Dingen Samuel gleich am Anfang das theokratische Moment in ganzer Schärfe hervorheben und gleich am Anfang den Versuch machen müssen, mit allem nur möglichen Nachdruck dem Volk ins Gewissen zu reden und ihm die religiöse Verwerflichkeit seines Begehrens zum Bewufstsein zu bringen. Und nun betrachten wir die Fortsetzung von c. 12, als welche c. 15 sich ergiebt. Konnte ein Erzähler, der eben erst berichtet hat, wie Jahve durch ein wunderbares Gewitter zur Erntezeit bekundet, dafs das Volk in seiner Wahl und Salbung des menschlichen Königs eine unverzeihliche Sünde begangen habe, konnte, frage ich, dieser Erzähler in dem nämlichen Athem fortfahren : „Samuel aber sprach zu Saul : Mich hat Jahve gesandt, dich zu salben zum König über sein Volk, über Israel* 15, 1 ? Konnte ein solcher 
15,11 und 35 schreiben? Hier ist mit neinem nur tragischen Contrast" S. 240 nichts geholfen, sondern es ist ein sachlicher Widerspruch. In dem ganzen c. 15 richtet sich die Verwerfung nicht auf die Sache, sondern lediglich auf die Person; es heifst nicht : Ich bereue es, einen König eingesetzt zu haben, wir wollen wieder zur reinen Theokratie zurückkehren, sondern : Ich bereue es, den Saul zum König eingesetzt zu haben, wir wollen deshalb einen Geeigneteren suchen. Der ganze Tenor von c. 15, von 10, 20-24, und auch, ich wiederhole es, von 8, 11-13 verträgt sich schlechterdings nicht mit einer principiellen Verwerfung jedes menschlichen Königthums als Abfall von der einzig gottgewollten Form der Theokratie, und so sehe ich mich genöthigt, doch bei meiner Quellenscheidung zu כאשר 8, 6 angesichts von Stellen wie Gen. 32, 3 E auf mir sitzen lassen mufs und deshalb dieses Argument nicht mehr so verwerthen darf - ich war zu jener unüberlegten Behauptung gekommen auf Grund der Empfindung, dafs es in diesem Sinne gewöhnlicher und correcter geheifsen haben würde ויהי כאשר אמרו ... וירע הרבר בעיני שמואל. $\mathrm{Ob}$ die sachlichen Bedenken, welche ich damals gegen die ursprüngliche Einheit von $M$ in seiner vorliegenden Gestalt geäufsert habe, wirklich so "nebensächlich" und unwesentlich sind, wie Budde sie werthet, möchte ich auch bezweifeln. Namentlich dafs Samuels richterliche Stellung und Würde in dieser ganzen Traditionsschicht erst später ein- und aufgetragen ist, mufs ich noch glauben. Der נביא der ältesten Tradition mufste erst zum I Sam. 9, 9) werden, ehe er zum שופט werden konnte : jene nothwenige Zwischenstufe des Samuel als נביא haben wir aber in dem von mir angenommenen Grundstock von M, den Samuel als שופט in der aufgezeigten Ueberarbeitung. Aber ich habe Budde doch zugestanden, dafs die Herkunft von ganz $\mathbf{M}$ aus $\mathbf{E}$ durch ihn erwiesen sei? Das 
führt mich auf eine Erörterung, welche uaber den Rahmen der Bücher Samuelis hinausgeht und ein allgemeineres Interesse beanspruchen darf. Nach meiner Ueberzeugung findet jenes Dilemma seine Lösung in einer Unterscheidung von $\mathrm{E}^{1}$ und $\mathrm{E}^{2}$. Budde selbst gesteht dies $\mathrm{S}$. 248 vollständig zu und erkennt auch S. 241 ausdrücklich „eine frühere Schicht" in $\mathbf{E}$ an , ohne den üblen Beigeschmack hoseanischer Betrachtung des Königthums." Dieser früheren Schicht nun gehört an, was ich in meiner ersten $\mathrm{Ab}$ handlung auf $\mathrm{E}$ zurückgeführt hatte, während mein vorjeremianischer Grundstock von Cap. 7 und 12 und meine "deuteronomistische" Ueberarbeitung von Cap. 8 u. 10, $17 \mathrm{ff}$. auf Grund der Budde'schen Beweisführung jetzt als $\mathrm{E}^{2}$ zu erscheinen haben, wofür ich vielleicht correcter $\mathrm{E}^{x}$ sagen würde, da ich weder behaupten und noch viel weniger beweisen kann, dafs dies gerade oder erst die zweite Hand war, welche an $\mathrm{E}$ thätig gewesen ist. Wenn Budde gegen diese Trennung einwendet, ${ }^{\text {dafs }}$ c. 7 und 12 sich in Stil und Eigenart von den übrigen Stücken durchaus nicht trennen lasse" S. 231, so berufe ich mich auf seine Worte an der schon einmal angeführten Stelle Jahrg. VII S. 161: "So heifst es zu viel verlangen von den alttestamentlichen Schriftstellern, dafs durchaus jeder einzelne von ihnen einen für heutige Forscher noch deutlich unterscheidbaren, ihm persönlich eigenthümlichen Stil geschrieben haben soll. Deshalb schiefsen meines Erachtens unter Andern Riehm und Dillmann über das Ziel hinaus, wenn sie nur da verschiedene Quellen zugeben wollen, wo ein verschiedener Stil bewiesen werden kann, und darum gegen $\mathrm{J}^{1} \mathrm{~J}^{2} \mathrm{~J}^{3}$, $\mathrm{P}^{1} \mathrm{P}^{2} \mathrm{P}^{3}$ u. s. w. Einspruch erheben. ${ }^{\alpha}$ In diesem „u. s. w. ${ }^{*}$ steckt sicherlich auch $\mathrm{E}^{1} \mathrm{E}^{2} \mathrm{E}^{3}$; auf jeden Fall ist für $\mathrm{E}^{1} \mathrm{E}^{2}$ $\mathrm{E}^{3}$ billig, was für $\mathrm{J}^{1} \mathrm{~J}^{2} \mathrm{~J}^{3} \mathrm{P}^{1} \mathrm{P}^{2} \mathrm{P}^{3}$ recht war. Was ich kurz als $\mathrm{E}^{2}$ bezeichnet habe und auch weiter bezeichnen werde, ist die letzte Hand, welche an $\mathrm{E}$ als selbstständigem Werk thätig gewesen ist und diese Quellenschrift zum 
Abschlusse gebracht hat. Und damit thun wir einen Einblick in ein hochwichtiges Vorkommnifs der hebräischen Literaturgeschichte. Um die Thatsache einer innerelohistischen Bearbeitung von $\mathrm{E}$ wahrscheinlich zu machen und dies $\mathrm{E}^{2}$ anderweitig nachzuweisen, berufe ich mich auf zwei Parallelen aus dem Richterbuch, welche Budde gleichfalls $\mathrm{E}$ zuschreibt. Die für unsern Fall wichtigste und bedeutsamste ist Jud. 8. Budde leitet diese ältere Gestalt der Gideonüberlieferung von $\mathrm{E}$ her, allerdings mit einem ${ }{ }^{\text {wahrscheinlich }}$ " S. 241. Gerade in diesem Capitel haben wir in der berühmten Stelle 8, 23 eine klassisch zu nennende Darstellung der Theokratie, welche jedes menschliche Königthum principiell ausschliefst. Aber auch hier beruht dieser Zug eben so sicher auf späterer Eintragung, wie in v. 27 die üble Deutung des goldenen Ephod; schon Studer hat richtig gesehen, dals nach dem ursprünglichen Sinn der Erzählung die Verwendung des erbeuteten Goldes zur Errichtung eines Ephod vielmehr zum Lob und Ruhm für Gideon gemeint war als ein Beweis von Uneigennützigkeit und Frömmigkeit, und dafs Gideon thatsächlich, wenn auch vielleicht nicht dem Namen nach, eine königliche Herrscherstellung einnahm, sagt Cap. 9 ganz ausdrücklich und schimmert auch in 8,30 ff. noch deutlich durch. Rührt also das Capitel Jud. 8 von $E$ her, so hätten wir auch hier ein $\mathbf{E}^{1}$ und $\mathbf{E}^{2}$ völlig analog mit dem von mir postulirten Verhältnifs von $\mathrm{E}^{1}$ und $\mathrm{E}^{2}$ in I Sam. 7-15. Weiterhin leitet Budde ausdrücklich von $\mathrm{E}$ her das Stück Jud. 6, 7-10 und zwar mit durchaus überzeugenden Gründen S. 232 Anm. Aber wenn irgend ein Stück in der Jud. 6, 1-8, 3 vorliegenden, vielfach überarbeiteten jüngeren Gestalt der Gideonüberlieferung den Eindruck eines nicht zum Organismus der ursprünglichen Erzählung gehörigen, rein äufserlichlich aufgelegten Flickens macht, so ist es 6,7-10. Angesichts dieser Thatsachen 
wird Budde auch in I Sam. 7-15 eine innerelohistische Ueberarbeitung im Princip gewifs zugeben.

Der von Budde erbrachte Beweis der Herkunft von I Sam. 7 u. 12 aus $\mathrm{E}$, wie ich genauer sage aus $\mathrm{E}^{2}$, hat nun aber eine weitergreifende Bedeutung; denn jene beiden Capitel nehmen eine ganz eigenartige Stellung ein, welche schon K. H. Graf richtig erkannt hat. Dieser macht nämlich darauf aufmerksam, dafs wir bier deutlich die Hand dessen wiedererkennen, nder die einzelnen Erzählungen aus der Richterzeit Richt. 2-16 unter eine einheitliche Geschichtsbetrachtung gebracht hat" (Gesch. Bücher des A. T. S. $97 \mathrm{f}$.) und hat hieraus schon den durchaus zutreffenden Schlufs gezogen, dafs die Samuelgeschichte ursprünglich zum Richterbuche gehört und I Sam. 12 dessen Abschlufs gebildet habe, ebenso wie das ganz analoge Capitel Jos. 24 den Abschlufs des Hexateuchs bildet. Unter den Beweisen für diese seine Behauptung führt Graf auch an die Parallelen I Sam. 7, 13 mit Jud. 3,30 [ich füge hinzu 4,23] 8,28 und 11, 33. Und in der That sind diese Stellen ganz besonders beweiskräftig; sie können nur von einer und derselben Hand geschrieben sein, denn die hier uns begegnende Verwendung der Wurzel כנy ist ganz einzigartig und hat auch in II Sam. 8, 1 nicht ein völlig entsprechendes Analogon. Diese Formel schliefst im Richterbuche die Erzählungen von den „grofsen" Richtern ab mit Ausnahme Simsons, bei welchem sie aus sachlichen Gründen schlechterdings nicht anzubringen war. Aber die Simsonsgeschichte weist, wie man das längst gesehen hat, in Jud. 13, 5 über sich selbst hinaus. Ihren Abschlufs bildet nach der ursprünglichen Absicht Saul, welcher vollendet, was Simson „angefangen ${ }^{“}$ hätte. Da tritt nun in I Sam. 7 Samuel an die Stelle Sauls. Samuel erscheint ganz wie die andern grofsen Richter auch; das Volk ist in schwerer Bedrängnifs durch äufsere Feinde, ein von Gott gesandter Helfer rettet es und 
„richtet" dann Israel. Und hier mache ich noch auf einen höchst beachtenswerthen Umstand aufmerksam, der gerade Samuel als wahren Erfüller des von Simson angefangenen Werkes besonders empfahl; die beiden Männer theilen nämlich - und zwar sie allein in der gesammten hebräischen Ueberlieferung - die Eigenschaft, schon vor ihrer Geburt geweihte Nasiraeer auf Lebenszeit zu sein, und so tritt denn der wahrhaft vom Geist Jahves erfüllte prophetische Nasiraeer Samuel an die Stelle dés in Fleischeslust und ungeistlichem Wesen untergegangenen Nasiraeers Simson. Es ist das Verdienst Stade's, in dieser Zeitschrift Jahrg. I S. 331 ff. zuerst das vordeuteronomische Richterbuch , in seinem letzten Grunde" ${ }^{\text {" }}$ von $\mathrm{E}$ hergeleitet zu haben : in diesen Formeln Jud. 3, 30. 4, 23. 8, 28. 11, 33. I Sam. 7, 13 haben wir recht deutlich des Merkmal einer sammelnden und redigirenden Hand, und der von Budde erbrachte Nachweis, dafs auch I Sam. 7 auf $\mathrm{E}$ zurückgeht, dient daher jenem Ergebnisse Stade's zur erwünschtesten Bestätigung. Dieses „vordeuteronomische Richterbuch“, wie 'es in der abschliefsenden Gestalt E's, also vorläufig kurz $\mathrm{E}^{2}$, erscheint, umfa/ste demnach die zwölf Richter Ehud, Debora-Barak, Gideon, Abimelech (in 10, 1 ausdrücklich mitgezählt), Thola, Jair, Jephtah, Ibzan, Elon, Abdon, Simson und Samuel. Dafs Thola, Jair, Ibzan, Elon und Abdon schon in diesem vordeuteronomischen Richterbuche standen, darf als sicher gelten, da Kuenen gewifs mit Recht in Jud. 10, 2. 5. 12, 9. 12. 15 die nämliche Hand erkennt, welche 12, 7 geschrieben hat (Onderzoek $^{2}$ I 342 u. 352), zu vergleichen auch 8,32 und 16, 31 . Nimmt man an der selbstständigen Zählung Abimelechs als ${ }_{n}$ Richter" einen, wie mir scheint durchaus begründeten, Anstofs, so gebe ich zu erwägen, ob nicht vielleicht auch Samgar schon dem vordeuteronomischen Richterbuch angehört hat : dann hätten wir, Abimelech nur als nothwendigen Anhang seines Vaters Gideon betrachtet, noch 
den weiteren Vortheil, dafs wir genau sechs, „kleine ${ }^{\alpha}$ (Samgar, Thola, Jair, Ibzan, Elon und Abdon) und sechs "grofse" ${ }^{4}$ Richter hätten : Ehud, Debora-Barak, GideonAbimelech, Jephtah, Simson und Samuel. Es ist dies eine sehr unsichere Vermuthung; denn gerade den nachgetragenen Samgar hält man für den allerspätesten Einschub, um an Abimelechs Stelle die Zwölfzahl der Richter vollzumachen, und ich verkenne die hierfür sprechenden Gründe (völlige Nichtberücksichtigung Samgars in 4, 1 und in dem chronologischen Fachwerk des gegenwärtigen Richterbuchs) nicht; aber Samgar ist doch durch das Deboralied zu gut beglaubigt und die That, welche von ihm berichtet wird, so charakteristisch, dafs ich mich nur im äufsersten Nothfalle dazu entschliefsen möchte, in diesem Vers einen ganz späten exilischen oder nachexilischen Lückenbülser zu sehen.

Doch ich kehre zu meinem eigentlichen Gegenstand zurück. Dafs I Sam. 7 Saul als glücklichen Philisterkämpfer und ersten nachdrücklichen Besieger dieses Erbfeindes, d. h. also Cap. 13 und 14 oder die Quelle G, nicht neben sich duldet, ist klar; stammt nun aber ganz $M$ aus $E$, so mufs ich einen Punkt meiner früheren Ausführungen modificiren. Ich hatte S. 129 die Vertheilung der Königswahl auf zwei Versammlungen, eine blofs vorbereitende zu Rama und eine definitive zu Mizpa, als harmonistische Ausgleichung von M mit $\mathrm{G}$ erklärt. Das darf ich jetzt nicht mehr. Aber auch hier hilft wieder $\mathrm{E}^{2}$. Dafs die Zerreifsung der Königswahl in zwei Versammlungen sachlich zu schweren Bedenken Anlafs giebt, mufs ich noch glauben - und spricht nicht die Erzählung 10, 20-24 für einen gewissermalsen improvisirten Charakter jener Versammlung? Wenn Samuel amtlich ganz Israel zu einem so wichtigen und entscheidenden Act zusammenberuft, war das Fehlen eines Mannes wie Saul selbst als Möglichkeit schwer zu verstehn. Aber wohl schon $\mathrm{E}^{2}$ 
fand diese von mir als ursprünglichen Sinn der Erzählung vorausgesetzte Handlungsweise zu „tumultuarisch", und gerade Cap. 7, wo wir sicher $\mathrm{E}^{2}$ haben, liefert hier wieder das völlige Analogon. Zuerst kommt Israel mit seinem Anliegen zu Samuel an einen nicht näher bezeichneten Ort und Samuel entbietet nun ,ganz Israel " nach Mizpa, um dort die Sache in optima forma zu erledigen. Dafs die Erwähnung von Mizpa allein noch kein Beweis für exilische oder nachexilische Abfassung ist, hat Budde S. 236 f. überzeugend nachgewiesen. Die einzelnen Punkte, welche Budde sonst noch zur Sprache gebracht hat, will ich auch wenigstens mit ein paar Worte berühren. Budde beanstandet S. 225, dafs auch in G Rama der Wohnort Samuels gewesen sei; aber Samuels Heimathsort ist doch Rama im Lande Zuph, und gerade im Lande Zuph liegt auch nach 9, 5 die "Stadt", in welcher Samuel wohnt. Dafs 9, 2 die Angabe über die Leibesgröfse Sauls ein späterer Eintrag aus 10, 23 sei S. 231, kann zugegeben werden. Höchst beachtenswerth urd gewifs richtig sind die Ausführungen S. $241 \mathrm{ff}$., dals das Fragment 10, 8 und 13, $7 \mathrm{~b}-15 \mathrm{a}$, in die Sondergeschichte der Fassung $\mathrm{G}^{\text {“ }}$ gehöre. Ueber 28, 3-25, welches Budde gleichfalls für G in Anspruch nimmt, mufs ich mich so lange des Urtheils enthalten, bis er die Mittelglieder aufgezeigt hat, stimme ihm aber von vorn herein im Princip zu : auch mir will es gar nicht in den Sinn, dafs diese herrliche und unschätzbare Quellenschrift mit 14, 51 völlig aufhöre, doch hat es bis jetzt nicht gelingen wollen, ihre Spuren weiter zu verfolgen. Möchte Budde uns doch möglichst bald seine mit Schmerzen erwartete Einleitung geben : sie wird gewifs - niemanden mehr interessiren als mich und ich werde mich gern von ihm überzeugen lassen.

Königsberg i. P. Neujahr 1890. 